\title{
Animal Experiment Study on Antitumor Effect of Matrine Liposome
}

\author{
JIANG Jianwei, WANG Chunlei and ZHANG Hongyan \\ Zhejiang Cancer Hospital, Zhejiang, 310022 China
}

\begin{abstract}
Objective: To study the antitumor effect of matrine liposome in mice. Methods: The mice were selected as the research object, and SPSS (statistic package for social science), matrine and matrine liposome were used for grouping. The antitumor effects of EAC, S180 and H22 were evaluated by tumor weight, thymus weight and spleen weight. Results: The inhibitory effect of matrine liposome on EAC, S180 and H22 in mice was significantly higher than matrine, $P<0.05$, which had statistical significance. Conclusion: Matrine liposome can effectively enhance the anti-tumor effect and improve the immunity of animals, which is worthy of clinical promotion.
\end{abstract}

Key words: Matrine, liposome, antitumor.

Matrine is an alkaloid extracted from plants, and is a combination of chemical structure, with a diuretic, dampness, anti-virus, anti-allergic, anti-tumor and immune function, very green, widely used in clinic. Liposome is one of the most commonly used anticancer drugs in clinic, which has the effects of long acting, targeting, stability, reducing dose and so on. In some sense, it can improve the anti-tumor targeting and reduce the side effects of chemotherapy. The combination of matrine and liposome can greatly enhance the anti-tumor effect. In this study, the mice were taken as the research object, and the inhibitory effect of matrine liposome on the tumor inhibitory effect was compared with the tumor inhibition rate.

\section{Materials and Methods}

\subsection{Experimental Data}

Invertebrate animals object: Select clean mice, half male and female, weighing about 22 g. Experimental drugs: matrine, matrine liposome. The matrine material was prepared by low concentration (0.5 $\mathrm{mg} / \mathrm{mL})$, medium concentration $(1 \mathrm{mg} / \mathrm{mL})$ and high concentration $(2 \mathrm{mg} / \mathrm{mL})$.

Corresponding author: Chunlei Wang, pharm D., research fields: Chinese medicine antitumor pharmacology.

\subsection{Experimental Method}

Research methods of EAC, S180, H22 tumor in mice: (1) Mouse S180 sarcoma ascites; (2) SPSS (statistic package for social science) (normal saline) dilution, the concentration of $1 \times 107 / \mathrm{mL}$; (3) Staining confirmed that the living cell rate reached 95\%; (4) $0.2 \mathrm{~mL}$ was given subcutaneously to the right anterior axillary of mice; (5) The mice were divided into control group (SPSS), matrine control group and matrine group. The drug group was divided into low dose group (25 mg/kg), middle dose group (50 mg/kg), high dose group (100 mg/kg), the drug group of mice by intraperitoneal injection of matrine liposome (1 day, 16d); (6) The mice were sacrificed and dissected to remove the spleen, tumor and thymus; (7) Wet weight, calculate tumor inhibition rate.

\subsection{Effect Standard}

The tumor weight, thymus weight and spleen weight were observed and recorded, and the tumor inhibition rate was used as the curative effect. Tumor inhibitory rate $=$ (Mean weight of tumor in model group - Mean weight of tumor in control group / mean weight of tumor in model group $\times 100 \%$. 


\subsection{Statistical Method}

Selecting SPSS 20.0 statistical software, measurement data $(x \pm s)$, the use of $t$ test, the comparison of clinical indicators between the groups, and setting $P<0.05$ for the difference were statistically significant.

\section{Results}

\subsection{Comparison of Antitumor Effect of EAC in Mice}

The experimental results show that under the same dosage, matrine liposome group inhibits EAC tumor effect, the matrine inhibitory rate was $50.23 \%$. Matrine liposome inhibition rate was $71.56 \%$, compared with $P$ less than 0.05 , with statistical significance, see Table 1.

\subsection{Comparison of Antitumor Effect of S180 in Mice}

The experimental results show that the low dose inhibition rate was $35.98 \%$, matrine and matrine liposomes could inhibit mouse S180 tumor, the middle dose group (54.98\%) tumor inhibition rate was the highest, compared with the same dose matrine group (32.56\%) $P<0.05$, with statistical significance; High dose group (35.98\%) compared with matrine group (32.56\%) $P>0.05$ shows no statistical significance.

\subsection{Comparison of Anti-tumor Effect of Mouse H22}

The experimental results showed that with the same dose, the rate of anti-tumor of Hishan in matrine group (56.8\%) and matrine liposome group (66.9\%) was lower than that in $P<0.05$, with statistical significance. The difference between matrine liposome group and CTX group $P>0.05$ see Table 3 for details.

\section{Discussion}

The clinical results showed that matrine had a significant effect on enhancing the anti-tumor effect. matrine can inhibit the proliferation of tumor cells, the differentiation of tumor cells, reversing tumor and other functions. Liposome, as a commonly used anticancer drug, has the characteristics of long effect, targeting, stability and reducing dosage, which

Table 1 Comparison of EAC indexes in mice.

\begin{tabular}{|c|c|c|c|c|c|c|}
\hline Group & $\mathrm{n}$ & Dosage (mg/kg) & Tumor weight (g) & Thymus weight (mg) & Spleen weight (mg) & Tumor inhibitory rate (\%) \\
\hline SPSS & 15 & - & $1.97 \pm 0.95$ & $28.97 \pm 7.52$ & $40.56 \pm 10.15$ & - \\
\hline Matrine & 15 & 50 & $0.99 \pm 0.27^{*}$ & $34.89 \pm 7.81^{*}$ & $42.93 \pm 7.68 *$ & 50.23 \\
\hline Matrine liposome & 15 & 50 & $0.55 \pm 0.45^{*}$ & $36.88 \pm 6.52^{*}$ & $47.91 \pm 9.84^{*}$ & 71.56 \\
\hline
\end{tabular}

Compared with NS group: * $P<0.05$.

Table 2 Comparison of antitumor effect of S180 in mice.

\begin{tabular}{|c|c|c|c|c|c|c|}
\hline Group & $\mathrm{n}$ & Dosage (mg/kg) & Tumor weight (g) & Thymus weight (mg) & $\begin{array}{l}\text { Spleen weight } \\
\text { (mg) }\end{array}$ & $\begin{array}{l}\text { Tumor inhibitory } \\
\text { rate }(\%)\end{array}$ \\
\hline SPSS & 15 & - & $2.35 \pm 0.89$ & $29.56 \pm 7.68$ & $39.31 \pm 6.95$ & - \\
\hline Matrine & 15 & 50 & $1.61 \pm 0.52 *$ & $35.26 \pm 6.34^{*}$ & $44.9 \pm 1.32$ & 32.56 \\
\hline Matrine liposome low-dose & 15 & 25 & $1.81 \pm 0.59$ & $32.98 \pm 9.97$ & $43.99 \pm 8.52$ & 35.98 \\
\hline Matrine liposome middle-dose & 15 & 50 & $1.13 \pm 0.63 * \Delta$ & $38.83 \pm 5.41^{* *}$ & $48.56 \pm 9.48^{*}$ & 54.98 \\
\hline Matrine liposome high-dose & 15 & 100 & $1.53 \pm 0.55^{*}$ & $36.99 \pm 7.39 *$ & $48.59 \pm 9.49$ & 23.27 \\
\hline
\end{tabular}

Compared with the NS group: $* P<0.05$, compared with matrine group $\Delta P<0.05$.

Table 3 Comparison of anti-tumor effect of mouse $\mathbf{H 2 2 .}$

\begin{tabular}{lllllll}
\hline Group & $\mathrm{n}$ & Dosage $(\mathrm{mg} / \mathrm{kg})$ & Tumor weight $(\mathrm{g})$ & Thymus weight $(\mathrm{mg})$ & Spleen weight (mg) & Tumor inhibitory rate (\%) \\
\hline SPSS & 15 & - & $2.35 \pm 0.21$ & $0.28 \pm 0.24$ & $42.52 \pm 9.97$ & - \\
CTX & 15 & 0.1 & $0.69 \pm 0.09$ & $0.26 \pm 0.09$ & $41.43 \pm 9.98$ & 68.51 \\
Matrine & 15 & 50 & $0.97 \pm 0.12^{*}$ & $0.35 \pm 0.27^{*}$ & $44.51 \pm 11.02^{*}$ & 56.8 \\
Matrine liposome & 15 & 50 & $0.75 \pm 0.09^{*}$ & $0.36 \pm 0.20^{*}$ & $49.67 \pm 11.38^{*}$ & 66.9 \\
\hline
\end{tabular}

Compared with NS group: * $P<0.05$. 
determines the necessity of enhancing the anti-tumor effect of matrine liposome. It can be seen from the experiment that the effect of matrine and matrine liposome on the anticancer effect of the same dose is significantly different, and it is very likely to be affected by liposome.

Data in Table 1 show that matrine liposome on mice EAC anti-tumor rate of up to $71.56 \%$, is significantly higher than matrine. Data in Table 2 show that the middle dose matrine liposomes inhibits S180 tumor of mice the best effect, the anti-tumor rate of $54.98 \%$, which is significantly higher than matrine. Data in Table 3 show that the same dose of matrine liposome $\mathrm{H} 22$ anti-tumor rate of mice is higher than matrine, $P<0.05$, with statistical significance. In addition, matrine liposome also has the role of enhancing the body's immunity, and the tumor weight of the mice in the matrine liposome group was negatively correlated with the thymus weight and spleen. The CTX group and the control group did not have this rule.

In summary, matrine liposomes can not only enhance the anti-cancer effect of animals, but also increase the body immunity, which is worth to be spread.

\section{References}

[1] Wu, C. H., Wang, H. F., and Song, F. 2009. "Transfection of Mouse Bone Marrow Mesenchymal Stem Cells with Lipofectamine-mediated Cytosine Deaminase.” Journal of Clinical Rehabilitative Tissue Engineering Research (01): 133-6.

[2] Li, H. S., Liu, X. P., Yu, P., Zou, J. Q., and Cui, Y. 2005. "Studies on Antitumor Effect of Matrine Liposome on Mice Transplanted Tumors." Chinese Archives of Traditional Chinese Medicine (10):1804-5.

[3] Zhang, Z. X., Wang, X. N., Yuan, F. T., and Zhou, D. Y. 2000. "Study on Activation of Immune Rejection after H-2K b Gene Transfection into Human Hepatoma Cells.” Chinese Journal of Cancer Biotherapy (02): 131-4.

[4] Huang, L. X., Xin, S. M., Ma, Z. Q., and Li, X. Y. 1992. "Regulation of Oleic Acid Polyphase Liposome (139) on Immune Responses in Mice.” Shanghai Journal of Immunology (06): 346-8+52.

[5] Wang, L., and Li, Q. L. 2009. "Research Progress on antitumor mechanism of Matrine.” Anhui Medical and Pharmaceutical Journal (04): 360-2.

[6] Shen, X. D., Song, G. B., Yan, R.B., and Yang, Y. 2005. "Research Progress of Matrine and Oxymatrine in the Anti-tumor Mechanism.” Journal of Chongqing University (Natural Science Edition) (06): 125-8.

[7] Wei, M., Liu, X. P., Li, H., Tang, Z. Y., and Li, H. S. 2006 "Study on Preparation of Matrine Liposome." Chinese Remedies \& Clinics (05): 328-30.

[8] Li, X. H., Xiao, M. H., Li, C. Q., and Li, X. Y. 2011. "Protective Effects of Matrine Liposome Nanoparticles on Immunological Liver Injury in Mice.” Journal of New Chinese Medicine (04): 118-9. 